Article

\title{
High-Resolution Hologram Calculation Method Based on Light Field Image Rendering
}

\author{
Xin Yang ${ }^{1,2}{ }^{\oplus}$, FuYang $\mathrm{Xu}^{3}$, HanLe Zhang ${ }^{1}$, HongBo Zhang ${ }^{4}$, Kai Huang ${ }^{3}$, Yong $\mathrm{Li}^{3, *}{ }^{3}$ and \\ QiongHua Wang ${ }^{1,2, *}$ \\ 1 School of Instrumentation and Optoelectronic Engineering, Beihang University, Beijing 100191, China; \\ holooptics@buaa.edu.cn (X.Y.); hanlezhang@stu.scu.edu.cn (H.Z.) \\ 2 Beijing Advanced Innovation Center for Big Data-based Precision Medicine, Beihang University, \\ Beijing 100191, China \\ 3 Institute of Information Optics, Zhejiang Normal University, Jinhua, Zhejiang 321004, China; \\ xfy@zjnu.cn (F.X.); kevin11132kevin@163.com (K.H.) \\ 4 Department of Computer and Information Sciences, Virginia Military Institute, Lexington, VA 24450, USA; \\ zhangh@vmi.edu \\ * Correspondence: liyong@zjnu.cn (Y.L.); qionghua@buaa.edu.cn (Q.W.)
}

Received: 14 December 2019; Accepted: 21 January 2020; Published: 23 January 2020

check for updates

Featured Application: The proposed method is applicable for static 3D advertising and holographic packaging.

\begin{abstract}
A fast calculation method for a full parallax high-resolution hologram is proposed based on elemental light field image (EI) rendering. A 3D object located near the holographic plane is firstly rendered as multiple EIs with a pinhole array. Each EI is interpolated and multiplied by a divergent sphere wave and interfered with a reference wave to form a hogel. Parallel acceleration is used to calculate the high-resolution hologram because the calculation of each hogel is independent. A high-resolution hologram with the resolution of $200,000 \times 200,000$ pixels is calculated within only eight minutes. Full parallax high-resolution 3D displays are realized by optical reconstructions.
\end{abstract}

Keywords: holographic 3D display; computer generated holography; light field image rendering; pinhole array

\section{Introduction}

Holography can fully reconstruct 3D scenes with all phase and amplitude information. Traditional optical holography can record very high-resolution holograms with lasers as illumination, but a stable optical platform is needed, as well as other optical devices. In contrast to optical holography, the 3D information can be coded into a computer-generated hologram for holographic 3D display [1,2]. Many efforts for dynamic holographic displays have been made for monochrome or color displays using lasers or light emitting diodes (LEDs) as illumination [3-8]. The progress on dynamic holographic 3D displays, however, still have proven difficult due to the constraints of limited bandwidth of the current available spatial light modulator (SLM) and huge amount of calculations.

High-resolution holograms such as rainbow holograms and Fresnel holograms are applicable for 3D advertising and packaging because of the emergence of holographic printing technology [9-14]. The biggest challenge for high-resolution holography is the huge amount of computation. Multiple algorithms such as point cloud-based, layer-based, and triangular mesh-based algorithms, as well as holographic stereogram methods have been proposed [15-18]. However, those methods are not effective for reducing the calculation burden for high-resolution holograms. We have proposed 
frequency fusing methods similar to holographic stereograms for full-parallax image hologram [11] and color rainbow hologram [10] calculations with high efficiency, but the sizes of the calculated holograms are limited by the RAM (Random Access Memory) of the computer. Rectangular tiling algorithms have been proposed for high-resolution hologram calculations free of limitations from computer RAM [19,20]. Taking Reference [19] as an example, each rectangular tile is calculated by using the 3D object data within the field of view (FOV) of this tile using the point cloud-based method. A full-parallax hologram with a resolution of $20,000 \times 20,000$ can take up to $32.9 \mathrm{~h}$. Another rectangular tiling-based algorithm is integral holography, which is the combination of integral imaging 3D displays and holography [21-24]. In this method, each element of the light field image (EI) rendered from a micro-lens array or pinhole array is firstly fast Fourier transformed (FFT) as the complex amplitude of each elemental hologram, also called a hogel. Then the hogel is calculated by the interference of the complex amplitude with a reference wave. However, the calculation of complex amplitude using FFT is time consuming, especially for large-scale high-resolution hologram calculation.

In order to reduce the computational time of the high-resolution hologram, and inspired by integral holography, a simple and fast calculation method without using FFT is proposed. A pinhole array is set behind the holographic plane for rendering EIs. Each EI located on the holographic plane is multiplied with a divergent spherical wave, which is regarded as the complex amplitude of this hogel. Additionally, a reference wave is interfered with complex amplitude for this hogel calculation. Parallel acceleration is used to speed up the calculation because the calculation of each hogel is independent. A full parallax high-resolution hologram with a resolution of $200,000 \times 200,000$ is calculated only within $8 \mathrm{~min}$. The validity of this proposed method is approved by optical reconstructions.

\section{Methods}

Figure 1 shows the rendering of EIs with a pinhole array for hologram calculation. PA is a pinhole array behind the holographic plane $\mathrm{H}$. The distance between PA and $\mathrm{H}$ is $f$. Obj is a 3D object in space, and $p(y, z)$ and $p_{2}\left(y_{2}, z_{2}\right)$ are two object points of obj. The EI is an image rendered from the 3D object to a pinhole. The $E I_{j}$, for example, is rendered from the pinhole $O_{j}$ by calculating the projected image from all 3D objects to $O_{j}$ within the opening angle from the pinhole to this EI. This $E I_{j}$ is coded into the Hogel $j$. In this situation, the size of EI is the same as the size of hogel, as well as their coordinates. The EI on the holographic plane has a width of $h$, and the FOV is dependent on the opening angle $\theta_{y}$ of the EI to the corresponding pinhole in the $y$ direction. Each EI is closely adjacent to other EIs. The coordinate of $j$-th pinhole $o_{j}$ is $y_{f j}$, and the projections of point $p(y, z)$ and $p_{2}\left(y_{2}, z_{2}\right)$ within the FOV to each pinhole are demonstrated. The intersections of projection lines to each pinhole on the holographic plane are coordinates of the projected points of EI. For example, in Figure 1, $y_{p j}$ is the coordinate of EI in the $y$ direction, which can be expressed as follows:

$$
y_{p j}=f \frac{y-y_{f j}}{z}+y_{f j}
$$

This calculation can also be applied to the $x$ direction, and the $x_{p i}$ of the projected EI can be expressed as follows:

$$
x_{p i}=f \frac{x-x_{f i}}{z}+x_{f i}
$$

where $(x, y, z)$ is the coordinate of the object point $P$ in the $3 \mathrm{D}$ coordinate system, and $\left(x_{p i}, y_{p j}\right)$ is the coordinate of EI $i m_{i, j}$ on the holographic plane. The coordinate of the $(i-t h, j-t h)$ pinhole is $\left(x_{f i}, y_{f j}\right)$. The $(i-t h, j-t h)$ EI can be expressed as follows:

$$
i m_{i, j}\left(x_{p i}, y_{p j}\right)=A_{p}
$$


where $A_{p}$ is the amplitude of object point $p(x, y, z)$ and $(i, j)$ is the index of the EI. The phase of this hogel on the holographic plane is a phase of the sphere wave from the pinhole to the hogel, which can be expressed as follows:

$$
\operatorname{phs}_{\mathrm{i}, \mathrm{j}}\left(x_{p i}, y_{p j}\right)=\frac{2 \pi}{\lambda}\left[\sqrt{\left(x_{p i}-x_{f i}\right)^{2}+\left(y_{p j}-y_{f j}\right)^{2}+f^{2}}\right]
$$

The complex amplitude of the object wave-front corresponding to each hogel is found by multiplying Equation (3) by a divergent sphere wave with the phase illustrated in Equation (4). This concept is simple and straightforward. From the view of the local area, the local amplitude and spherical wave control the amplitude and propagation direction of this small beamlet.

Assuming that the reference light is a plane wave, and the angle between the plane wave in the $y$ direction and the $z$ axis is $\theta_{r e f y}$, the off-axis amplitude type $(i-t h, j-t h)$ hogel can be expressed as follows:

$$
H_{i, j}\left(x_{p i}, y_{p j}\right)=i m_{i, j}\left(x_{p i}, y_{p j}\right) \cos \left[p h s_{i, j}\left(x_{p i}, y_{p j}\right)-\frac{2 \pi}{\lambda} y_{p j} \sin \left(\theta_{r e f y}\right)\right]
$$

After the parameters of the PA and FOV of the hologram, as well as other necessary parameters are provided, each hogel can be calculated independently. All the hogels form the final high-resolution hologram.

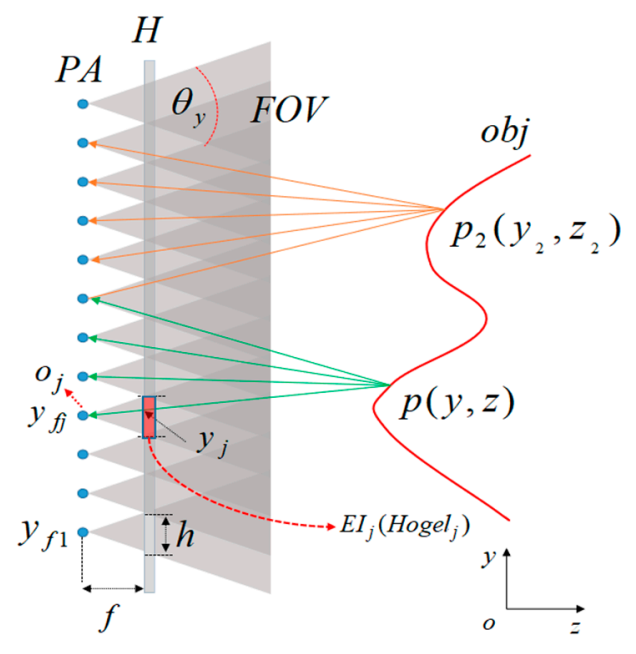

Figure 1. The rendering of elemental light field images (EIs) with a pinhole array for hologram calculation.

\section{Experiment and Results}

The pixel pitch $d_{h}$ of the hologram is $0.318 \mu \mathrm{m}$, which is determined by our holographic printer [9-11]. This principle of the home-made holographic printer is similar to the holographic printer introduced in Reference [12], but the light source in our holographic printer is a blue LED with a center wavelength of $365 \mathrm{~nm}$ rather than a laser. The maximum frequency that the holographic printer can support is $\frac{1}{2 d_{h}}=1573$ line/mm. Printing holograms at the highest resolution requires extremely stability for the printing system. In practical usage, almost half of the highest resolution is used for hologram design. In the experiment, the diffraction angles of the FOV in the $x$ and $y$ directions are set as $\theta_{x}$ is $48^{\circ}$, and $\theta_{y}$ is $20^{\circ}$, respectively. The angle of the reference wave in the $y$ direction and $z$ axis is set as $22^{\circ}$, assuming the wavelength is $632 \mathrm{~nm}$. Therefore, the max frequency is $\frac{\sin \left(\theta_{x} / 2\right)}{\lambda}=643 \mathrm{line} / \mathrm{mm}$ in the $x$ direction, and the max frequency is $\frac{\sin \left(\theta_{y} / 2\right)}{\lambda}+\frac{\sin \left(\theta_{\text {refy }}\right)}{\lambda}=867$ line/mm in the $y$ direction, respectively. This is assuming that the distance between the pinhole and holographic plane is $f=$ $1 \mathrm{~mm}$. In this situation, the width and length of each hogel or EI is $w=2 f \tan \left(\frac{\theta_{x}}{2}\right)=0.89 \mathrm{~mm}$ and $h=2 f \tan \left(\frac{\theta_{y}}{2}\right)=0.35 \mathrm{~mm}$. The geometric relationship of the hologram calculation using a 3D point 
cloud model with two layers is shown in Figure 2a. The first layer has a distance of $10 \mathrm{~mm}$ while the second layer has a distance of $20 \mathrm{~mm}$ from the holographic plane $\mathrm{H}$. The two-layer Chinese characters are shifted in the vertical direction to guarantee not to overlap when they are viewed from the front view for simplicity. The 3D model has a size of $64 \times 64 \times 10 \mathrm{~mm}(\mathrm{~W} \times \mathrm{H} \times \mathrm{D})$ with $984 \mathrm{~K}$ object points. The front view of the 3D model is shown in Figure $2 b$.

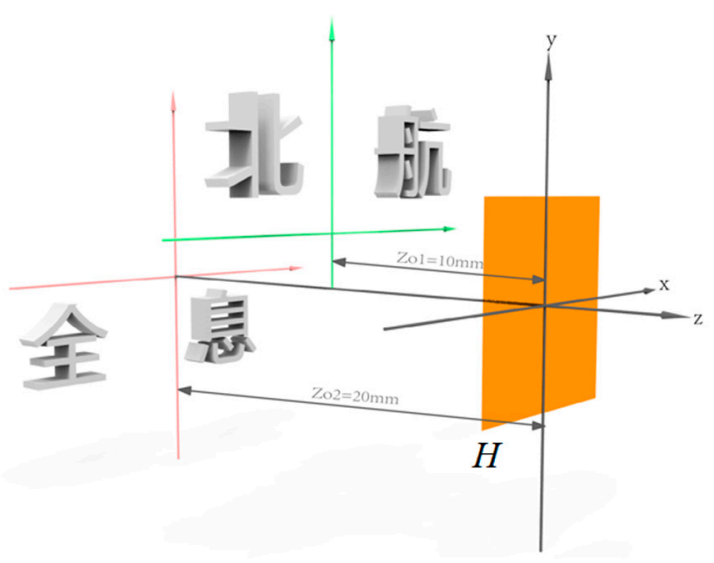

(a)

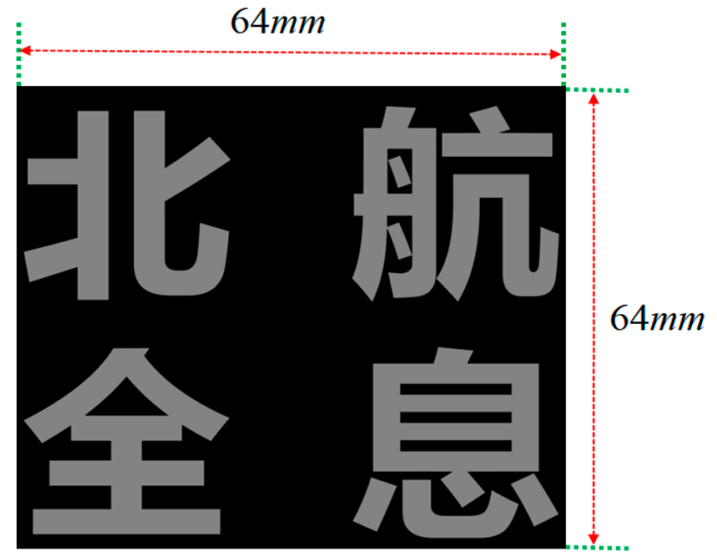

(b)

Figure 2. The geometric relationship for hologram calculation: (a) the side view of the holographic plane $\mathrm{H}$ and 3D model; (b) the front view of the 3D model. The first layer Chinese characters are the abbreviation of "Beihang" University and the second layer Chinese characters mean "Holography".

In the rendering of the EIs, the sampling intervals for both $x$ and $y$ directions are set as $4 \mu \mathrm{m}$ and the resolution of each EI is $222 \times 88$ pixels. The parameters for hologram calculation are summarized in Table 1. In the proposed method, the size of the EI is the same as the size of the hogel, but the sampling intervals are different. After we get an EI from the 3D model, this EI is firstly interpolated to a new EI with the same resolution as the hogel. The interpolated EI is used for hologram calculation according to Equation (5). In the calculation, after the size of the EI is determined, the number of the EI is calculated by $63.6 / 0.89=71.46 \approx 71$ and $63.6 / 0.35=181.71 \approx 181$. The resolution $200 \mathrm{~K} \times 200 \mathrm{~K}$ of the hologram is also an approximation of the physical size of $63.6 \times 63.6 \mathrm{~mm}$. The approximation is possible because missing a little bit of data on the outer edge of the hologram does not affect the 3D display.

Table 1. Parameters for hologram calculation.

\begin{tabular}{cc}
\hline Parameters & Values \\
\hline Number of points in the 3D model & $984 \mathrm{~K}$ \\
Size of hologram & $63.6 \times 63.6 \mathrm{~mm}$ \\
Resolution of hologram & $200 \mathrm{~K} \times 200 \mathrm{~K}$ \\
Pixel pitch of hologram & $0.318 \mu \mathrm{m}$ \\
Resolution of hogel & $2800 \times 1108$ pixels \\
Wavelength & $632 \mathrm{~nm}$ \\
Size of EI & $0.89 \times 0.35 \mathrm{~mm}$ \\
Pixel pitch of EI & $4 \mu \mathrm{m}$ \\
Resolution of EI & $222 \times 88$ pixels \\
Number of EIs & $71 \times 181$ \\
\hline
\end{tabular}

Figure 3a shows the rendered light field image, which contains all EIs rendered from the 3D model. Figure $3 b$ is an enlarged part of the light field image. The light field image almost has a resolution of $16 \mathrm{~K} \times 16 \mathrm{~K}$, which is much higher than the resolution of current commercially available display panels. The high-resolution light field image and high-resolution hologram guarantee high imaging quality and a large FOV of reconstructed 3D images. 


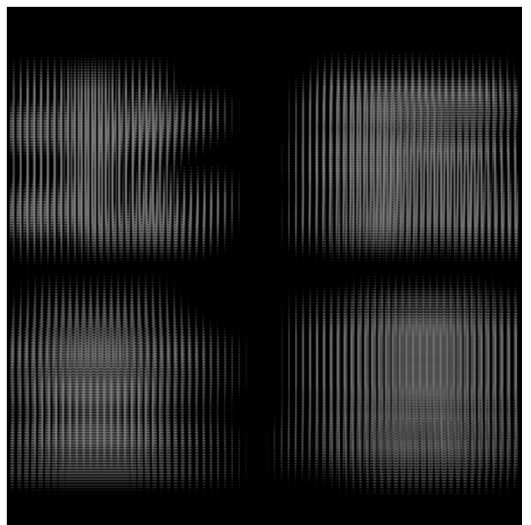

(a)

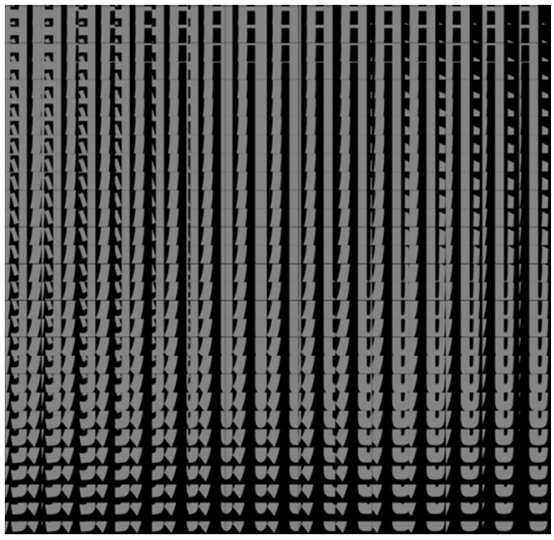

(b)

Figure 3. The rendered light field image: (a) light field image; (b) enlarged part of (a).

Because of the independent property of calculations for each hogel in the proposed method, "parfor" in MATLAB is used for parallel acceleration. MATLAB 2015b and a laptop (i7-9750HQ CPU, 16G RAM and 1024G SSD) were used for hologram calculations. Six CPU cores were used in the hologram calculation. The computational time was about $8 \mathrm{~min}$ for the calculation of this hologram, including the time used for EI rendering. The hologram was printed with our home-made holographic printer from the Institute of Information Optics, Zhejiang Normal University, in about $4.5 \mathrm{~h}$.

The overall diagram of the holographic printer is shown in Figure 4. The light from the LED is filtered and collimated to illuminate the digital micro mirror (DMD) with a resolution of $1024 \times 768$ pixels. A small portion of the calculated hologram with a resolution of $600 \times 600$ pixels is first zero padded to the same resolution as the DMD and then loaded into the DMD for display. The modulated light from the DMD is imaged on the photosensitive material through the objective lens, and this small portion of the hologram is printed. Controlled by the computer, the motion platform is moved to the next position to print the next hologram section. With this holographic printer, the maximum size (a $200 \times 200 \mathrm{~mm}$ hologram with pixel pitch of $0.318 \mu \mathrm{m}$ ) can be printed on a photoresist plate. The part of the calculated hologram and the printed hologram are shown in Figure 5a,b, respectively.

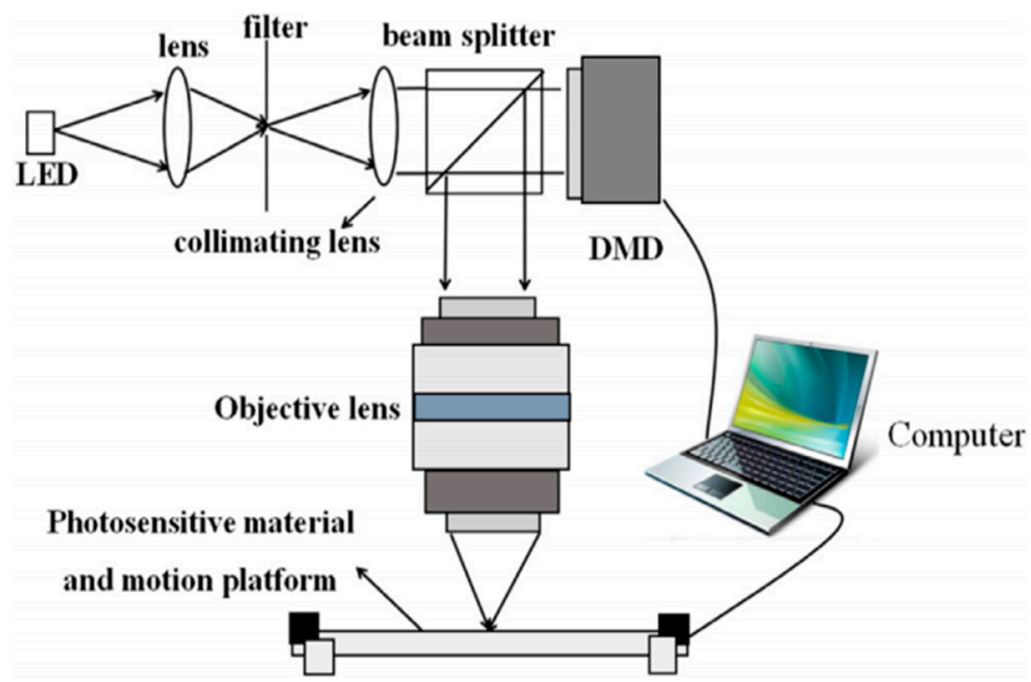

Figure 4. The diagram of holographic printer. 


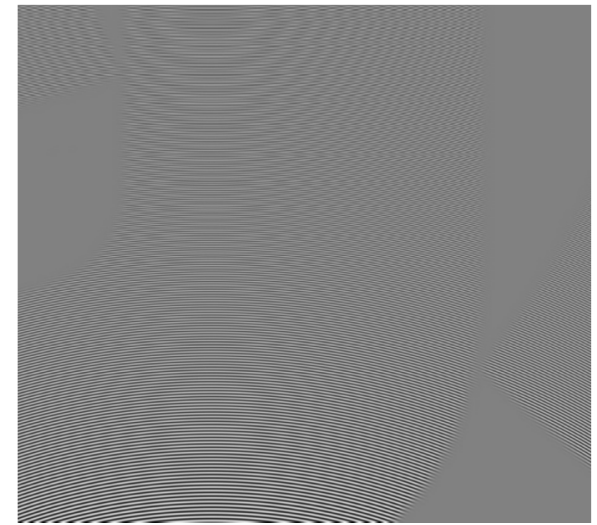

(a)

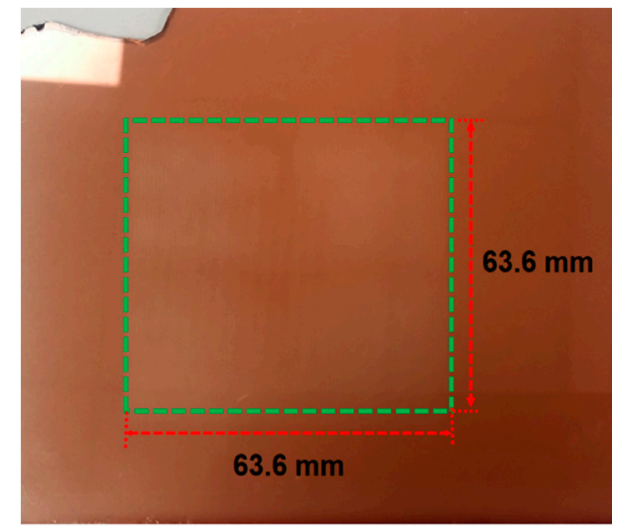

(b)

Figure 5. Holograms for 3D display: (a) part of the calculated hologram; (b) printed hologram.

In the reconstruction, a halogen lamp available in our lab is used as illumination source. The reconstruction of the hologram is demonstrated in Figure 6a. The white light is coupled into the fiber and a divergent light from the fiber head illuminates the hologram $\mathrm{H}$ with a proper incline angle in the $y$ direction. The diffracted light propagates into the human eye for watching the reconstructed 3D image. The real image in front of the hologram plane or the virtual image behind the hologram plane can be watched by selecting a proper illumination angle. Figure $6 \mathrm{~b}-\mathrm{d}$ show three reconstructed images from three different viewing angles. Video S1 shows the reconstruction from different viewpoints. 


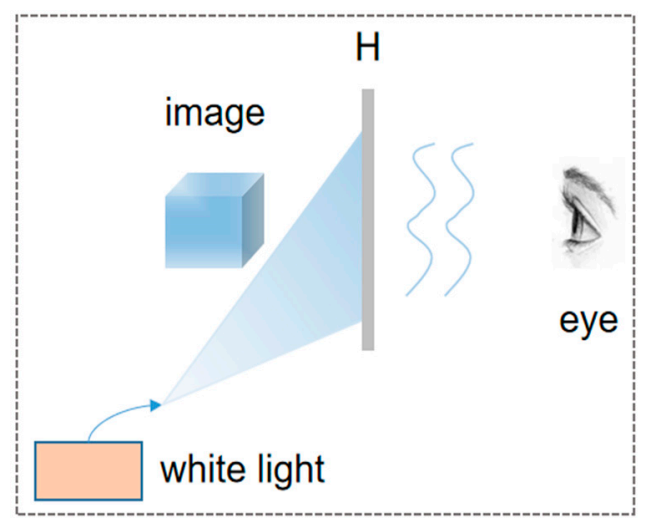

(a)

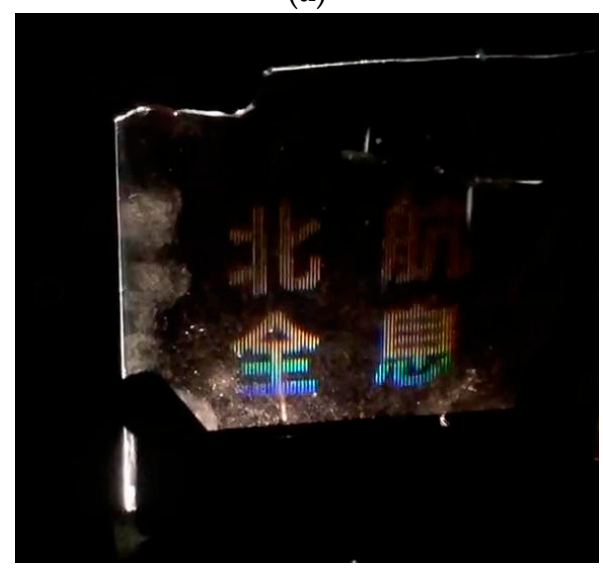

(c)

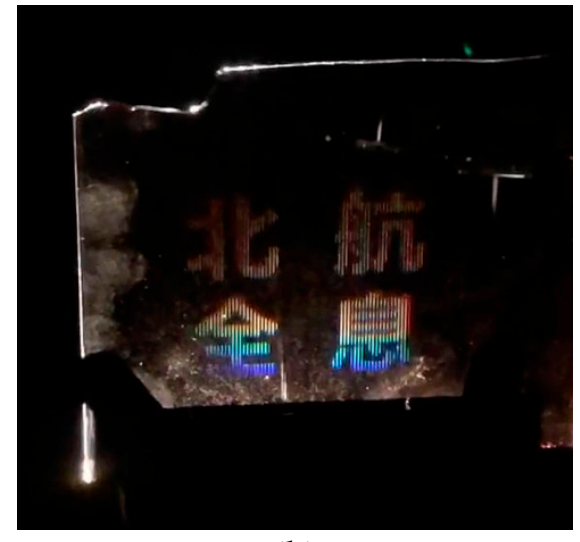

(b)

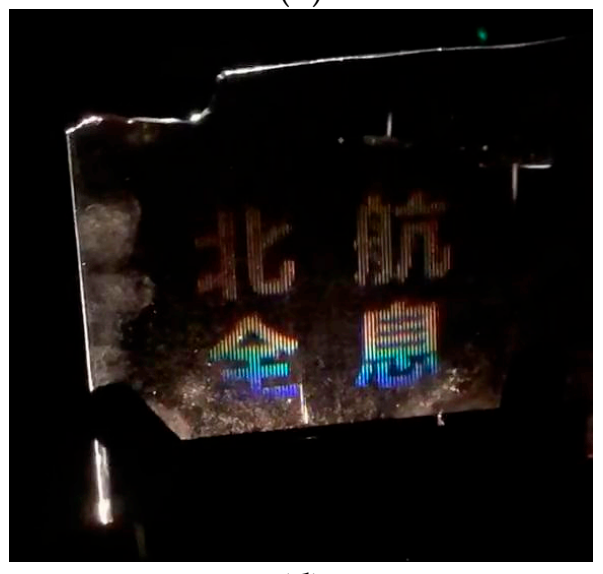

(d)

Figure 6. (a) The diagram of optical setup for reconstruction; (b-d) different views of the reconstructed 3D image. Video S1 shows the reconstruction from different viewpoints.

From the optical reconstruction, the parallax is smooth and the FOV is large. The brightness is a little different due to the uneven incline illumination on this large-area hologram. Figure 7 shows three images captured by our camera, focusing on part of the holographic plane with different viewing angles. The two speckles in the red and green circles on the holographic plane are used as reference positions. From the results we can know that different perspectives of light come from different positions on the holographic plane, which is the core point of our method to encode the propagation of each pixel in the EI to different angles with divergent sphere wave phase factors.

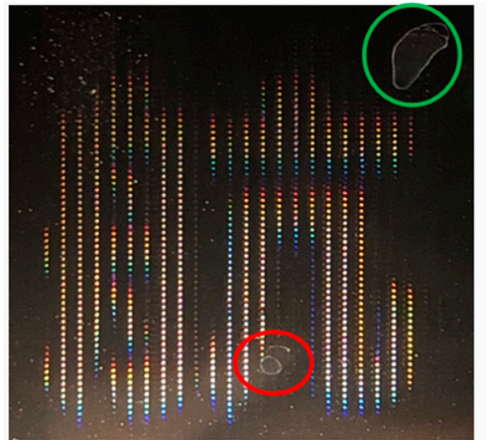

(a)

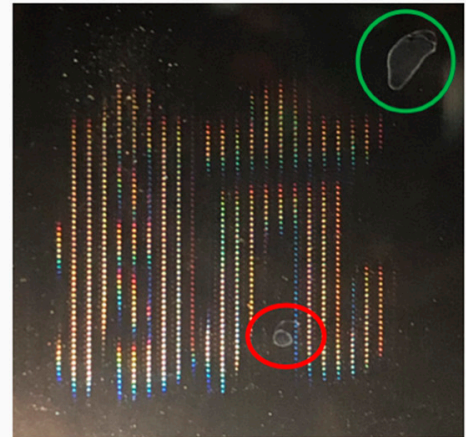

(b)

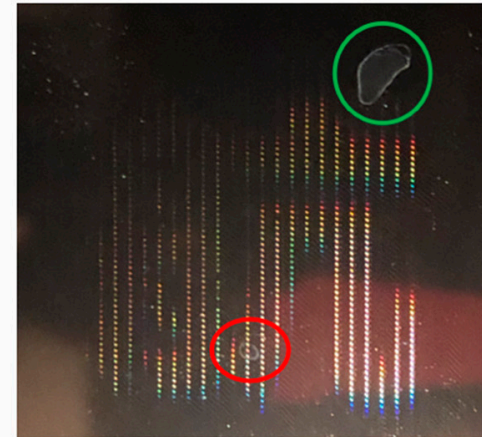

(c)

Figure 7. (a-c) Three different images captured by the camera focused on the holographic plane. 
The large hogel size in the first experiment means that human eyes can easily distinguish each hogel on the holographic plane. The 3D image, viewed by the human eye, is comparable to watching through a fence. In order to reduce the fence effect and improve the quality of the displayed result, the second experiment is designed and carried out using the same model as the first experiment, but with different parameters. In the second experiment, the distance between the pinhole array and the holographic plane is $f=0.25 \mathrm{~mm}$. In this situation, each hogel has a resolution of $700 \times 277$ pixels, corresponding to the physical size of $0.22 \times 0.09 \mathrm{~mm}$. The sampling pitch in each EI is set as $1 \mu \mathrm{m}$ and the resolution of each EI is $222 \times 88$ pixels, which is the same as in the first experiment. The number of EIs is $287 \times 725$. The resolution of the full light field image is about $63.7 \mathrm{~K} \times 63.7 \mathrm{~K}$ in this experiment. Other parameters are all the same as the parameters of the first experiment. The calculation time of this hologram is approximately $37 \mathrm{~min}$. Figure 8 shows the three reconstructed images. Video $\mathrm{S} 2$ shows the $3 \mathrm{D}$ reconstruction from different viewing angles.

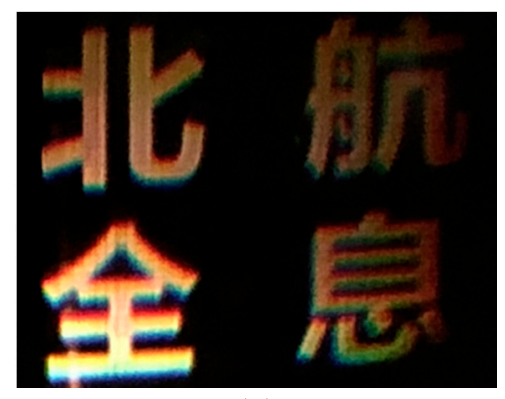

(a)

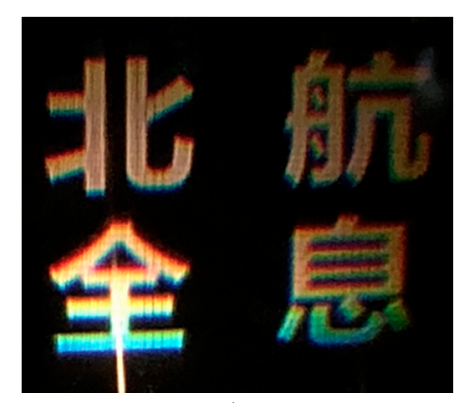

(b)

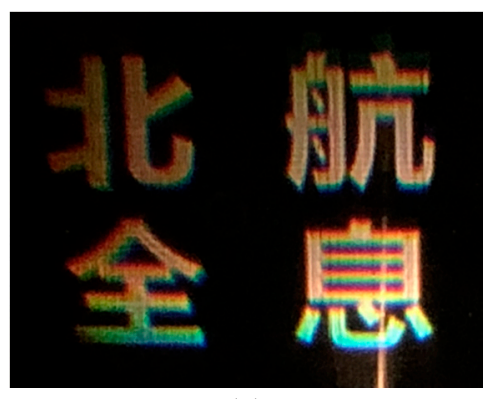

(c)

Figure 8. $(\mathbf{a}-\mathbf{c})$ Three reconstructed images from different viewpoints. Video $\mathrm{S} 2$ shows the reconstruction from different viewpoints.

\section{Discussion}

Encoding the high-resolution light field image into a high-resolution hologram has two important advantages: displaying 3D images with higher resolutions, and dramatically decreased calculation times of high-resolution holograms. In our proposed method, a pinhole array behind the holographic plane is used for the EI's rendering, and a divergent sphere wave is used as the phase information for each EI. However, the pinhole array can also be set in front of the holographic plane, and the convergent sphere wave can be used as phase information.

In the calculation, converting each EI to the complex amplitude information on each hogel plane using FFT is not needed in the proposed method. Additionally, the phase factor of divergent sphere waves is the same for all hogels, which means the phase factor only needs to be calculated one time and can be reused for each hogel calculation. This direct encoding method can greatly reduce the computational time of high-resolution holograms.

In the encoding process of holograms, a plane wave is used as a reference wave for simplicity. Actually, a divergent sphere wave is more practical, because the white light we used (or a white light LED) for illumination of the hologram is approximately a divergent wave. The reason why a white light can be used to reconstruct the hologram is that there is minimal distance between the reconstructed image and the holographic plane, similar to the case of the image holography, the color dispersion due to the white light illumination does not have a large influence on the reproduced 3D image. It should be noted that to display the hologram with laser as illumination is still possible with a higher resolution of reconstructed 3D images, but speckle noise will exist.

We demonstrate two experiments for 3D display with different hogel size and sampling pitch. From the results, we can conclude that the calculation time is associated with the number of object points in the 3D model and EIs, as well as the sampling interval in each EI. Well-designed parameters will guarantee high-quality reconstructed images with a relative short computational time. Parallel computing using a GPU (graphics processing unit) is still possible for further reduction of computational time. 
The displayed results with white light as illumination are a grayscale 3D display. However, the concept of full color rainbow holography $[9,12]$ can also be applied to our method for a full color $3 \mathrm{D}$ display at the cost of losing vertical parallax.

In the proposed method, the resolution of each EI and the size of the EI or hogel are related, which means a large FOV will cause large hogel sizes and lead to more of the fence effect. A large-depth 3D display is not possible with this method because an image far from the holographic plane will be blurred due to the white light illumination. However, the proposed method is still valuable for holographic packing or 3D display purposes with relatively small depth.

\section{Conclusions}

In this study, a simple and fast method for full parallax high-resolution holographic 3D display is demonstrated without using FFT. The encoding of the high-resolution light field image with the holographic method to speed up the calculation is the essence of our method. A high-resolution hologram with 40 billion pixels is calculated only within eight minutes, and the optical 3D reconstruction is effectively approved by experiments.

Supplementary Materials: The following are available online at http://www.mdpi.com/2076-3417/10/3/819/s1, Video S1: holographic reconstruction with large hogel size in the first experiment, Video S2: holographic reconstruction with small hogel size in the second experiment. The codes are used to calculate the hologram in the second experiment. The modified parameters can be used for the calculation of the hologram in the first experiment with the same codes.

Author Contributions: X.Y. wrote the program for hologram calculation and completed the relevant experiments; H.Z. and F.X. rendered the light field images and printed the hologram; H.Z. helped to discuss the research and modified the document; K.H. helped to prepare data for printing; Y.L. and Q.W. led the project and provided support. All authors have read and agreed to the published version of the manuscript.

Funding: This research was funded by National Key R\&D Program of China, under Grant No. 2017YFB1002900 and by National Natural Science Foundation of China under Grant No. 61927809.

Conflicts of Interest: The authors declare no conflict of interest.

\section{References}

1. Yaras, F.; Kang, H.; Onural, L. State of the art in holographic display: A survey. J. Disp. Technol. 2010, 6, 443-454. [CrossRef]

2. Matsushima, K.; Arima, Y.; Nakahara, S. Digitized holography: Modern holography for 3D imaging of virtual and real objects. Appl. Opt. 2011, 50, H278-H284. [CrossRef] [PubMed]

3. Yang, X.; Zhang, H.; Wang, Q.H. A fast-computer-generated holographic method for VR and AR near-eye 3D display. Appl. Sci. 2019, 9, 4164. [CrossRef]

4. Lin, S.; Wang, D.; Wang, Q.; Kim, E. Full-color holographic 3D display system using off-axis color-multiplexed-hologram on single SLM. Opt. Laser Eng. 2020, 126, 105895. [CrossRef]

5. Maimone, A.; Georgiou, A.; Kollin, J. Holographic near-eye displays for virtual and augmented reality. ACM Trans. Graph. 2017, 36, 8501-8516. [CrossRef]

6. Li, X.; Liu, J.; Zhao, T.; Wang, Y. Color dynamic holographic display with wide viewing angle by improved complex amplitude modulation. Opt. Express 2018, 26, 2349-2358. [CrossRef]

7. Gao, H.; Xu, F.; Liu, J.; Dai, Z.; Zhou, W.; Li, S.; Yu, Y.; Zheng, H. Holographic three-dimensional virtual reality and augmented reality display based on $4 \mathrm{~K}$-spatial light modulators. Appl. Sci. 2019, 9, 1128. [CrossRef]

8. Lin, S.; Cao, H.; Kim, E. Single SLM full-color holographic three-dimensional video display based on image and frequency-shift multiplexing. Opt. Express 2019, 27, 15926-15942. [CrossRef]

9. Shi, Y.; Wang, H.; Li, Y.; Jin, H.; Ma, L. Practical method for color computer generated rainbow holograms of real-existing objects. Appl. Opt. 2009, 48, 4219-4226. [CrossRef]

10. Yang, X.; Wang, H.; Li, Y.; Xu, F.; Zhang, H.; Zhang, J. Large scale and high resolution computer-generated synthetic color rainbow hologram. J. Opt. 2019, 21, 025601. [CrossRef]

11. Yang, X.; Wang, H.; Li, Y.; Xu, F.; Zhang, H.; Zhang, J.; Yan, Q. Computer generated full-parallax synthetic hologram based on frequency mosaic. Opt. Commun. 2019, 430, 24-30. [CrossRef] 
12. Yamaguichi, T.; Yoshikawa, H. High resolution computer generated rainbow hologram. Appl. Sci. 2018, 8, 1955. [CrossRef]

13. Kunieda, O.; Matsushima, K. High-quality full-parallax full-color three-dimensional image reconstructed by stacking large-scale computer-generated volume holograms. Appl. Opt. 2019, 58, G104-G111. [CrossRef] [PubMed]

14. Yamamoto, Y.; Nakayama, H.; Takada, N.; Nishitsuji, T.; Sugie, T.; Kakue, T.; Shimobaba, T.; Ito, T. Large-scale electroholography by HORN-8 from a point-cloud model with 400,000 points. Opt. Express 2018, 26, 34259-34265. [CrossRef]

15. Su, P.; Cao, W.; Ma, J.; Cheng, B.; Liang, X.; Cao, L.; Jin, G. Fast computer-generated hologram generation method for three-dimensional point cloud model. J. Disp. Technol. 2016, 12, 1688-1694. [CrossRef]

16. Arai, D.; Shimobaba, T.; Murano, K.; Endo, Y.; Hirayama, R.; Hiyama, D.; Kakue, T.; Ito, T. Acceleration of computer-generated holograms using tilted wavefront recording plane method. Opt. Express 2015, 23, 1740-1747. [CrossRef]

17. Liu, J.; Liao, H. Fast occlusion processing for a polygon-based computer-generated hologram using the slice-by-slice silhouette method. Appl. Opt. 2018, 57, A215-A221. [CrossRef]

18. Abookasis, D.; Rosen, J. Three types of computer-generated hologram synthesized from multiple angular viewpoints of a three-dimensional scene. Appl. Opt. 2006, 45, 6533-6538. [CrossRef]

19. Zhang, H.; Zhao, Y.; Cao, L.; Jin, G. Fully computed holographic stereogram based algorithm for computer-generated holograms with accurate depth cues. Opt. Express 2015, 23, 3901-3913. [CrossRef]

20. Blinder, D.; Shimobaba, T. Efficient algorithms for the accurate propagation of extreme-resolution holograms. Opt. Express 2019, 27, 29905-29915. [CrossRef]

21. Zhang, H.; Deng, H.; Li, J.; He, M.; Li, D.; Wang, Q.H. Integral imaging-based 2D/3D convertible display system by using holographic optical element and polymer dispersed liquid crystal. Opt. Lett. 2019, 44, 387-390. [CrossRef]

22. Zhang, Y.; Fu, Y.; Wang, H.; Li, H.; Pan, S.; Du, Y. High resolution integral imaging display by using a microstructure array. J. Opt. Technol. 2019, 86, 100-104. [CrossRef]

23. Ai, L.; Cao, H.; Sun, H.; Shi, X. Performance enhancement of integral imaging based Fresnel hologram capturing by the intermediate view reconstruction. Opt. Express 2019, 27, 31942-31955. [CrossRef] [PubMed]

24. Zhang, X.; Lv, G.; Wang, Z.; Hu, Z.; Ding, S.; Feng, Q. Resolution-enhanced holographic stereogram based on integral imaging using an intermediate-view synthesis technique. Opt. Commun. 2020, 457, 124656. [CrossRef] 